\title{
Surfaces of prescribed Weingarten curvature tangential to a cone
}

\author{
James N. Holland
}

\begin{abstract}
In this paper we investigate the existence and regularity of solutions to a Dirichlet problem for a Hessian quotient equation on the sphere. The equation arises as the determining equation for the support function of a convex surface which is required to meet a given enclosing cone tangentially and whose $k$ th Weingarten curvature is a prescribed function $\psi$. This is a generalization of a related problem treated in [7] and is motivated by results from the theory of curvature flows $[16,17]$. In the general case, we are able to obtain $C^{1}$ estimates provided $\psi$ satisfies a certain weak asymptotic growth condition. Under further regularity assumptions we are able to demonstrate, via a priori estimates and the continuity method, the existence of bounded $C^{2, \alpha}$ solutions under a convexity condition on $\psi$. We also demonstrate conditions under which no solution can exist.
\end{abstract}

\section{Introduction and problem formulation}

In this paper we treat the problem of finding a convex surface $M$, whose boundary is tangential to the boundary $\partial \tilde{C}$ of a given cone $\tilde{C} \subset \mathbb{R}^{n+1}$, with convex cross-section and vertex at the origin, and whose $k$ th Weingarten curvature, $S_{k}(\kappa(M))$, is a prescribed function $\psi$. Here $\kappa(M)$ are the principal curvatures of $M, S_{k}$ is the $k$ th elementary symmetric polynomial and $\psi=$ $\psi(x, u, \nu)$, where $\nu=\nu(x, u)$ is the unit normal to $\operatorname{graph}(u)$ at $(x, u)$. We prove the existence of such a surface under some assumptions on $\psi$. In particular, we are interested in the following problem:

Problem 1. Given a cone $\tilde{C} \subset \mathbb{R}^{n+1}$ with vertex at the origin and a $C^{3, \alpha}$ uniformly convex cross-section, with slope along $\partial \tilde{C}$ in direction $\eta$ determined by the function $K(\eta)>0, k<n$ and a strictly positive function $\psi(x, z, p) \in C^{2, \alpha}\left(\mathbb{R}^{n} \times \mathbb{R}^{+} \times \overline{S_{-}^{n}}\right)$ then find a region $\Omega^{*} \subset \mathbb{R}^{n}$, possibly 
unbounded, and a strictly convex function $u: \Omega^{*} \rightarrow \mathbb{R}$ such that

$$
S_{k}(\kappa(\operatorname{graph}(u))=\psi(x, u, \nu)
$$

holds in $\Omega^{*}$ and the boundary conditions

$$
\begin{aligned}
\left|D_{\eta} u\right| & =K(\eta), \\
u(x) & =\tilde{C}(x)
\end{aligned}
$$

hold on $\partial \Omega^{*}$ where $\eta=x /|x|$. These conditions should be interpreted as limits holding as $|x| \rightarrow \infty$ in those directions in which $\Omega^{*}$ is unbounded. Further investigate the regularity of $\Omega^{*}, u$.

This boundary condition can be interpreted as the surface graph $(u)$ being required to meet tangentially with the enclosing boundary $\partial \tilde{C}$. The motivation in considering problems of this sort comes from the theory of curvature flows where a central item of study is the existence and behaviour of complete, non-compact surfaces which move by either translation or homothety, the so-called self-similar flows. Significant progress has been achieved in the case of surfaces which move by mean curvature or by a power of their Guass curvature. In particular, in [16] the author demonstrates that any complete hypersurface $M$ moving homothetically by a positive power of its Gauss curvature is contained in some convex cone with vertex at the origin $M \subset C$ and further that $M$ is asymptotic to $\partial C$. Conversely, it is demonstrated that given any such cone $C$ and any positive power $\alpha$ (in an appropriate range), there exists a complete, non-compact hypersurface $M \subset C$ asymptotic to $\partial C$ such that $M$ moves homothetically under its Gauss curvature to the power $\alpha$. In a similar spirit in [12] it is demonstrated that any complete, non-compact $C^{2}$ hypersurface with bounded gradient moving homothetically under mean curvature flow is again contained within, and asymptotic to, a unique convex tangent cone. In attempting to investigate results of this sort for more general Weingarten curvatures determined by the elementary symmetric polynomials of the principal curvatures, one naturally runs into problems analogous to Problem 1.1. In the first section we introduce an assumption on $\psi$ which will guarantee that $\Omega^{*}$ is bounded. An asymptotic growth condition is natural and we introduce the following rather weak condition. 
Assumption 1. There exists $C>0, \beta<k, R>0$ such that

$$
\psi(x, u, \nu) \geq C|x|^{-\beta}
$$

outside the ball $B_{R} \subset \mathbb{R}^{n}$ uniformly in $u$ and $\nu$.

Under this assumption we are able to prove the following:

Theorem 1. If $\psi \in C^{2, \alpha}\left(\mathbb{R}^{n} \times \mathbb{R}^{+} \times \overline{S_{-}^{n}}\right)$ satisfies Assumption 1 and is everywhere strictly positive then there exists a constant $C$, depending only on $\psi$ and the cone $\tilde{C}$, such that any convex $C^{2}$ solution $u$ of Problem 1 satisfies

$$
\begin{aligned}
\|u\|_{C^{1}} & \leq C, \\
\operatorname{diam}\left(\Omega^{*}\right) & \leq C .
\end{aligned}
$$

In obtaining higher estimates for equations of the form (1.1) the function $\psi$ would typically be assumed to satisfy some sort of convexity condition. In particular, because we intend to treat Problem 1 via the support function of $\operatorname{graph}(u)$ it would be standard to assume that $\psi^{-\frac{1}{k}}$ is convex in the pair $(x, u)$ and strictly convex in $x[3,4,7]$. Such a convexity assumption is used only in reducing the interior $C^{2}$ estimate (for the support function) to a boundary estimate and is essentially necessary. The natural class of surfaces in which to seek a solution to Problem 1 is the class of $k$-convex surfaces. However, since we will treat the problem using the support function associated with a convex hypersurface it becomes necessary to restrict ourselves to considering only convex solutions. Examples constructed in [1] demonstrate that this requires the imposition of an additional assumption. In $[5,6]$ the authors investigate various assumptions sufficient to ensure that the solution is convex. From their work it becomes clear that the simplest and most natural assumption able to ensure convexity of the solution is convexity of $\psi^{-1 / k}$ in $(x, u)$. However, such a convexity assumption would be incompatible with the growth condition (1.3) except in the case where $\psi$ was independent of $x$. As a preliminary result we hence only obtain a complete $C^{2, \alpha}$ estimate in the case $\psi=\psi(\nu)$, however, this will later be extended in Theorem 4 and Corollary 1. We have:

Theorem 2. If $\psi=\psi(\nu) \in C^{2, \alpha}\left(\overline{S_{-}^{n}}\right)$ is strictly positive then there exists a solution $\left(u, \Omega^{*}\right)$, with $u \in C^{3, \alpha}\left(\Omega^{*}\right)$ strictly convex, $\Omega^{*}$ bounded and with $C^{2, \alpha}$ boundary, to Problem 1. 
Problem 1 is similar in many respects to the problem treated in our earlier work [7] where we considered the problem of the existence of a surface of prescribed curvature over a fixed region $\Omega$ satisfying the alternative boundary condition

$$
\lim _{x \rightarrow \partial \Omega}|D u(x)|=\infty,
$$

which can be interpreted as the surface graph $(u)$ meeting an enclosing cylinder tangentially. The problem in [7] was a generalization of the previously studied case of Gauss curvature to more general curvatures. The treatment of Problem 1 is in many ways analogous to the treatment in [7]; however, the calculations are more complicated as Problem 1 exhibits a richer range of behaviours than that considered in [7]. We introduce, as in [10], the support function $h$ of the convex surface $\operatorname{graph}(u)$ defined on the lower unit hemisphere $S_{-}^{n}$ by

$$
h(\nu)=\sup \{(x, u(x)) \cdot \nu \quad \text { for }(x, u(x)) \in \operatorname{graph}(u)\} .
$$

It is well known that $\operatorname{graph}(u)$ can be uniquely recovered from $h$ as the appropriate boundary component of

$$
\left\{x \in \mathbb{R}^{n+1} \mid x \cdot \nu \leq h(\nu) \quad \forall \nu \in \bar{S}_{-}^{n}\right\} .
$$

Furthermore we have, as in [8], the expression

$$
\nabla h(\nu)=(x, u(x))-h(\nu) \nu
$$

where $\nabla h$ represents the covariant gradient of $h$ on the sphere and the point $(x, u(x)) \in \operatorname{graph}(u)$ has normal $\nu$. Problem 1 is then equivalent to the following problem for the support function $h$ of the hypersurface $\operatorname{graph}(u)$ :

Problem 2. Given $k<n$, and a uniformly convex $C^{3, \alpha}$ region $\Omega \subset S_{-}^{n}$ with $\operatorname{dist}\left(\Omega, \partial S_{-}^{n}\right)>0$ find a function $h: \Omega \rightarrow \mathbb{R}$ such that

$$
S_{n, n-k}^{\frac{1}{k}}\left(\lambda\left(\nabla^{2} h+h I\right)\right)=f(\nu, h, \nabla h)
$$

is satisfied on $\Omega$ subject to the conditions $h \equiv 0$ on $\partial \Omega$ and $\left(\nabla^{2} h+h I\right)>0$. Here we set

$$
\begin{aligned}
& S_{n, n-k}^{\frac{1}{k}}\left(\lambda\left(\nabla^{2} h+h I\right)\right):=\frac{S_{n}^{\frac{1}{k}}\left(\lambda\left(\nabla^{2} h+h I\right)\right)}{S_{n-k}^{\frac{1}{k}}\left(\lambda\left(\nabla^{2} h+h I\right)\right)}, \\
& f(\nu, h, \nabla h):=\psi^{-\frac{1}{k}}(\nabla h-h(\nu) \nu, \nu)=\psi^{-\frac{1}{k}}((x, u), \nu) .
\end{aligned}
$$


The operator $\nabla^{2}$ represents the covariant Hessian on the sphere and $\lambda\left(\nabla^{2} h+\right.$ $h I)$ represents the set of eigenvalues of $\left(\nabla^{2} h+h I\right)$.

As we will be working on the sphere, when we treat the support function $h$, we will denote $\nu=x$ so that we have $f=f(x, h, \nabla h)$. The identically zero Dirichlet boundary condition arises naturally, in light of the boundary condition that $\operatorname{graph}(u)$ meets $\partial \tilde{C}$ tangentially, when one considers the geometric definition of the support function on the sphere at point $\nu$ as the signed distance from the origin to the tangent hyperplane of the surface at the point with normal $\nu$. The convexity of $\Omega$ is implied by the convexity of $u$. To see this observe that, taking the standard stereographic projection of the sphere onto the plane $\left\{x_{n+1}=-1\right\}$, the region $\Omega$ becomes $D u\left(\Omega^{*}\right)$ and the Legendre transform of $u$, denoted $u^{*}$, can be written as $u^{*}\left(x^{\prime}\right)=\zeta h\left(\zeta^{-1} x^{\prime},-\zeta^{-1}\right)$ where $\zeta=\left(1+\left|x^{\prime}\right|^{2}\right)^{1 / 2}$ and $x^{\prime}=\left(x_{1}, \ldots, x_{n}\right)$. Since $u^{*} \equiv 0$ on $\partial D u\left(\Omega^{*}\right)$ and $u^{*}$ is convex this implies that $D u\left(\Omega^{*}\right)$ is convex and subsequently its preimage under stereographic projection, the domain $\Omega$, is also convex. From Equation (1.4) one observes that the estimate $\|u\|_{C^{0}\left(\Omega^{*}\right)} \leq C$ implies the estimate $\|h\|_{C^{1}(\Omega)} \leq C$ and so Theorem 2 follows immediately from Theorem 1 and the following estimate:

Theorem 3. Let $f(x, z, p) \in C^{2, \alpha}\left(\overline{S_{-}^{n}} \times \overline{\mathbb{R}}^{+} \times \mathbb{R}^{n+1}\right)$ be strictly positive, convex in $(z, p)$ and strictly convex in $p$. Then, if one has the estimate $\|h\|_{C^{1}} \leq C$, there exists a globally $C^{3, \alpha}$ solution $h$ to Problem 2.

To illustrate the way in which the asymptotic behaviour of $\psi$ in $x$ is crucial to the behaviour of the problem we provide the following non-existence result:

Theorem 4. For any cone $\tilde{C}$ there exists a constant $C_{0}=C_{0}(\tilde{C})$ such that for any strictly positive $\psi$ satisfying

$$
\psi(x, u, \nu) \leq C_{0}|x|^{-k}
$$

Problem 1 has no solution, neither bounded nor unbounded. Furthermore, there exists an additional constant $C_{1}=C_{1}(\tilde{C})$ such that given any strictly positive function $\psi$ satisfying

$$
\psi(x, u, \nu) \geq C_{1}|x|^{-k}
$$

outside of some ball, there exists a $C^{3, \alpha}$ solution to Problem 1 provided that $\psi^{-1 / k}$ is convex in $(x, u)$ and strictly convex in $x$. 
The existence component of Theorem 4 is a straightforward corollary of Theorem 3 and a slight modification of the proof of Theorem 1 and its proof will not be given. The non-existence result in Theorem 4 is essentially optimal. We cannot extend the exponent in (1.6) to be less than $k$ as this would contradict with Theorems 2 and 3 since one could construct a $\tilde{\psi}$ satisfying both (1.6) and the conditions of those theorems. Furthermore, direct calculation in the radially symmetric case shows that we cannot allow the constant $C_{0}$ to be independent of the cone $\tilde{C}$. From Theorem 4 we have:

Corollary 1. Given any cone $\tilde{C}$ of uniformly convex cross-section and any $C^{3, \alpha}$ function $\psi(x, u, \nu)>0$, convex in the pair $(x, u)$ and strictly convex in $x$, for which there exists constants $C, \epsilon$ and $R$ such that

$$
\epsilon|x|^{-k} \leq \psi \leq C|x|^{-k}
$$

uniformly in $(u, \nu)$, where the first inequality holds for $x$ outside $B_{R}$, then there exists $a \lambda^{*}>0$ such that Problem 1 with the cone $\tilde{C}$ and

$$
S_{k}(\kappa(\operatorname{graph}(u)))=\lambda \psi
$$

has a bounded convex solution for $\lambda>\lambda^{*}$ and no solution, either bounded or unbounded for $\lambda<\lambda^{*}$.

Proof. From Theorem 4 we deduce that there exists $\lambda_{1}^{*}>0$ and $\lambda_{2}^{*}>0$ such that Equation (1.7) has no solution for $\lambda<\lambda_{1}^{*}$ and a convex bounded solution for $\lambda>\lambda_{2}^{*}$ and it remains only to demonstrate that we may take $\lambda_{1}^{*}=\lambda_{2}^{*}$. However, defining $\lambda^{*}$ as the infimum of $\lambda$ such that Equation (1.7) has a solution we see, by definition, that for any $\lambda>\lambda^{*}$ there exists a $\underline{\lambda}<\lambda$ such that Equation (1.7) has a solution for $\lambda=\underline{\lambda}$. Direct calculation shows that this solution can be used as a subsolution to the problem for $\lambda$ after transforming to the problem for the support function on the sphere and so one may derive the appropriate $C^{2}$ estimates as outlined in later sections and the result follows.

The question of what happens when $\lambda=\lambda^{*}$ is of particular interest. In general, one may have a bounded solution, no solution, a complete noncompact solution or a solution bounded in some directions but unbounded in others. Complete non-compact hypersurfaces of prescribed curvature are of particular interest due to their connections with solutions to the curvature flow equations which move by translation or homothety. However, one must impose very strong conditions on $\psi$ to ensure that at $\lambda=\lambda^{*}$ any solution 
would be a complete and non-compact hypersurface asymptotically tangential to the cone $\tilde{C}$. This can be seen by considering the support function $h$ of the hypersurface. We would require $h$ to simultaneously satisfy both the Dirichlet boundary condition $h \equiv 0$ on $\partial \Omega$ and the "blow-up" condition $|\nabla h| \rightarrow \infty$ as one approaches $\partial \Omega$. As seen in $[7,15]$, in general, it is not possible to prescribe both conditions simultaneously. In the case in which both $\tilde{C}$ and $\psi$ are rotationally symmetric one may explicitly write the required conditions on $\psi$.

Theorem 5. Let $\psi=\psi(|x|)$ be $C^{3, \alpha}$ and the cone $\tilde{C}$ have circular cross section and slope $K$ and define the function

$$
d(R)=k \int_{0}^{R} r^{n-1} \psi(r) d r-\left(\frac{K}{\sqrt{1+K^{2}}}\right)^{k}\left(\begin{array}{l}
n-1 \\
k-1
\end{array}\right) R^{n-k} .
$$

There exists a rotationally symmetric, bounded, $C^{3, \alpha}, k$-admissible solution $u$ to Problem 1 if and only if there exists an $0<R^{*}<\infty$ such that $d\left(R^{*}\right)=0$ and $d(R)<0$ for all $0<R<R^{*}$. Furthermore, there exists a complete, noncompact, $k$-convex solution to Problem 1 if and only if $d(R)<0$ for all $R>0$ and

$$
\int_{0}^{\infty}(g(s)-K)>-\infty
$$

where the function $g$ is given by

$$
g(s)=\frac{\left(k \int_{0}^{s} r^{n-1} \psi(r) d r\left(\begin{array}{c}
n-1 \\
k-1
\end{array}\right)^{-1} s^{k-n}\right)^{1 / k}}{\left(1-\left(k \int_{0}^{s} r^{n-1} \psi(r) d r\left(\begin{array}{c}
n-1 \\
k-1
\end{array}\right)^{-1} s^{k-n}\right)^{2 / k}\right)^{1 / 2}} .
$$

In each case the solution will be convex if and only if the function $g(s)$ is non-decreasing on the range over which $u$ is defined.

The layout of the remainder of the paper is as follows. In Section 4 we obtain the proof of Theorem 2 by proving Theorem 3. In Section 2 we obtain an estimate on sup $|u|$ which corresponds to a global $C^{1}$ estimate for the support function $h$ by following the ideas introduced in [7]. In Section 3 we obtain a global $C^{2}$ estimate on $h$. In Section 3.1 we reduce the global $C^{2}$ estimate of $h$ to the boundary following standard procedures. Finally in 
Section 4 we employ the standard arguments of the continuity method and the established a priori bounds to obtain our existence result. In Section 4 we also prove Theorem 5 and the non-existence component of Theorem 4 .

\section{2. $C^{1}$ estimate}

We obtain a global $C^{1}$ estimate on $h$ by obtaining instead an estimate on $\sup |u|$ which is well known to be equivalent. We first obtain an estimate for $|u(0)|$ and

$$
|u(\underline{x})|=\min _{x \in \Omega^{*}}|u(x)| .
$$

We may assume $u(\underline{x}) \geq 0$. Let $\tilde{C}_{A}$ denote the cross-section of the cone $\tilde{C}$ at height $A$. By the convexity of the cross-sections the cross-section $\tilde{C}_{1}$ contains a ball $B_{r} \subset \mathbb{R}^{n}$ for some fixed $r>0$. Therefore the cross-section $\tilde{C}_{u(\underline{x})}$ will contain the ball $B_{u(\underline{x}) r}$. We have, as in [13], for a general $k$-convex body $\Omega$ and a general $k$-convex function $g$, the following formula:

$$
\int_{\Omega} S_{k}(\kappa(\operatorname{graph}(g))) \leq C \int_{\partial \Omega} S_{k-1}(\kappa(\partial \Omega)),
$$

where the constant $C$ depends only on $n, k$ and $\left(\sup _{\Omega}|D g|\right)$. Applying this formula to the ball $B_{u(\underline{x}) r}$ contained in the cross-section $\tilde{C}_{u(\underline{x})}$, we derive

$$
\int_{B_{u(\underline{x}) r}} \psi=\int_{B_{u(\underline{x}) r}} S_{k}(\kappa(\operatorname{graph}(u))) \leq C \int_{\partial B_{u(\underline{x}) r}} S_{k-1}\left(\kappa\left(\partial B_{u(\underline{x}) r}\right)\right)
$$

noting that the convexity of $\operatorname{graph}(u)$ and the boundary condition (1.2) imply that $\sup _{B_{u(0) r}}|D u| \leq \sup _{\mathbb{S}^{n}} K<\infty$. We may compute directly the right-hand side of expression (2.1). Doing so we obtain

$$
\int_{\partial B_{u(\underline{x}) r}} S_{k-1}\left(\kappa\left(\partial B_{u(\underline{x}) r}\right)\right)=C(u(\underline{x}) r)^{n-k}
$$

for some new constant $C$ which has absorbed several factors dependant on $n$ and $k$. Coupling this with our asymptotic growth estimate on $\psi$ we observe $\epsilon C_{1}(u(\underline{x}) r)^{n-\beta}-C_{2} \leq \epsilon \int_{B_{u(\underline{x}) r} \backslash B_{R}}|x|^{-\beta} \leq \int_{B_{u(\underline{x}) r} \backslash B_{R}} \psi \leq C(u(\underline{x}) r)^{n-k}-C_{0}$, where $R$ is the radius in Assumption 1 and $\epsilon$ is the constant appearing in Assumption 1. Here $C_{0}$ depends on $R$ and $k$. We may also assume that $u(\underline{x}) r>R$. Taking the limit as $u(\underline{x}) \rightarrow \infty$ leads to a contradiction and 
we therefore obtain the bound $u(\underline{x}) \leq C$ for some constant $C$ depending only on $n, \tilde{C}, k$ and the asymptotic behaviour of $\psi$. This bound implies a corresponding bound $|\underline{x}| \leq C_{2}$ given that $u \geq \tilde{C}$ everywhere. We hence have $|u(0)| \leq|u(\underline{x})|+C_{2} \sup K \leq C_{3}$. We now proceed from this estimate to establish the complete $\sup _{\Omega^{*}}|u|$ estimate. We formulate the statement in the following theorem:

Theorem 6. Let $h: \Omega \rightarrow \mathbb{R}$ be a globally $C^{3, \alpha}$ solution to Problem 2. Then, provided $\psi, \tilde{C}$ satisfy the conditions in Theorem 1, there exists a constant $C$ depending only on $k, n, \tilde{C}$ and $\psi$ such that

$$
|h|+|\nabla h| \leq C
$$

on $\bar{\Omega}$.

Proof. As previously noted this follows from an estimate on $\sup _{\Omega^{*}}|u|$. We proceed as in [7]. We first suppose that $\Omega^{*}$ is bounded and hence $\sup _{\Omega^{*}}|u|<$ $\infty$. Consider the point $x_{0} \in \partial \Omega^{*}$ such that $\left|x_{0}\right|=\max \left\{|x|: x \in \partial \Omega^{*}\right\}$ and set $H=u\left(x_{0}\right)$, as noted we are considering solutions in which $\partial \operatorname{graph}(u) \subset$ $\partial \tilde{C}$ and hence $\left(x_{0}, H\right) \in \partial \tilde{C}$. We set $R=\left|x_{0}\right|=H / K\left(x_{0} /\left|x_{0}\right|\right)$ and denote $\zeta=x_{0} /\left|x_{0}\right|$ for convenience. We note $K$ is bounded below by a positive constant. We consider the point $x^{*}$ in $\Omega^{*}$ given by $x^{*}=(R-1) \zeta$, assuming w.l.o.g. that $R>1$. We have the corresponding points on $\partial \tilde{C}$ and $\operatorname{graph}(u)$ given by $\left(x^{*},(R-1) K(\zeta)\right)$ and $\left(x^{*}, u\left(x^{*}\right)\right)$, respectively, which we denote as $\partial \tilde{C}\left(x^{*}\right)$ and $\operatorname{graph}(u)\left(x^{*}\right)$. We desire first an estimate for the difference $\epsilon=u\left(x^{*}\right)-(R-1) K(\zeta)>0$. Elementary convex geometry implies that the derivative $D_{\zeta} u\left(x^{*}\right)$ satisfies the conditions

$$
\left|D_{\zeta} u\left(x^{*}\right)\right|+u\left(x^{*}\right)<H
$$

and

$$
u\left(x^{*}\right)-(R-1)\left|D_{\zeta} u\left(x^{*}\right)\right| \leq C,
$$

where $C$ is the estimate on $u(0)$. Therefore writing $D_{\zeta} u\left(x^{*}\right)=K(\zeta)-\delta$ we obtain that

$$
\epsilon \leq \delta
$$

and

$$
\epsilon+\delta\left(\frac{H}{K(\zeta)}-1\right) \leq C
$$


so that in turn

$$
\epsilon \leq \frac{K(\zeta) C}{H}=\frac{C}{R}
$$

and $\epsilon$ is estimated in terms of $R$. The "gap" between $u$ and the cone $\tilde{C}$ can be bounded in other directions as well. In particular, defining

$$
\epsilon(\eta)=u((R-1) \eta)-\tilde{C}((R-1) \eta)
$$

for $\eta \in S^{n-1}$ provided $u((R-1) \eta)$ is defined and $\epsilon(\eta)=0$ if $u((R-1) \eta)$ is not defined we have, by the above reasoning, $\sup _{\eta \in S^{n-1}} \epsilon(\eta) \leq \frac{C}{R}$. We now employ the technique in [7] together with Assumption 1. Consider that component of $\partial \tilde{C}$ whose projection onto the plane forming the first $n$ coordinates lies within the annulus $A \subset \mathbb{R}^{n}$ of outer radius $R$ and inner radius $R-1$ we denote this component by $\tilde{C}_{R}$. We deform this component to obtain a new surface $C$ given as the graph of some function $G$ with

$$
G=|x| \phi\left(\frac{x}{|x|}\right)+\Psi_{\epsilon}(|x|),
$$

where $\phi$ is the function on $S^{n-1}$ whose degree one homogeneous extension determines the cone $\tilde{C}$ and $\Psi_{\epsilon}$ represents the perturbation to $\tilde{C}$ which yields $C$. We may calculate the first- and second-order derivatives of $G$ directly. We have

$$
\begin{aligned}
G_{i}= & \frac{x_{i}}{|x|} \phi(\eta)+|x|\left(\sum_{k} \phi_{k}(\eta)\left(\frac{\delta_{i k}}{|x|}-\frac{x_{i} x_{k}}{|x|^{3}}\right)\right)+\frac{x_{i}}{|x|}\left(\Psi_{\epsilon}\right)^{\prime}(|x|), \\
G_{i j}= & \frac{x_{i} x_{j}}{|x|^{2}}\left(\Psi_{\epsilon}\right)^{\prime \prime}(|x|)+\left(\Psi_{\epsilon}\right)^{\prime}(|x|)\left(\frac{\delta_{i j}}{|x|}-\frac{x_{i} x_{j}}{|x|^{3}}\right)+\phi(\eta)\left(\frac{\delta_{i j}}{|x|}-\frac{x_{i} x_{j}}{|x|^{3}}\right) \\
& +\frac{x_{i}}{|x|} \sum_{k} \phi_{k}(\eta)\left(\frac{\delta_{k j}}{|x|}-\frac{x_{k} x_{j}}{|x|^{3}}\right)+\frac{x_{j}}{|x|} \sum_{k} \phi_{k}(\eta)\left(\frac{\delta_{i k}}{|x|}-\frac{x_{k} x_{i}}{|x|^{3}}\right) \\
& +|x| \sum_{k, m} \phi_{k m}(\eta)\left(\frac{\delta_{i k}}{|x|}-\frac{x_{i} x_{k}}{|x|^{3}}\right)\left(\frac{\delta_{m j}}{|x|}-\frac{x_{m} x_{j}}{|x|^{3}}\right) \\
& +|x| \sum_{k} \phi_{k}(\eta)\left(\frac{3 x_{i} x_{k} x_{j}}{|x|^{5}}-\frac{x_{i} \delta_{j k}+x_{k} \delta_{i j}}{|x|^{3}}-\frac{\delta_{i k} x_{j}}{|x|^{3}}\right),
\end{aligned}
$$

where we have denoted

$$
\eta=\frac{x}{|x|}
$$


From this we immediately observe the following estimates:

$$
\left|G_{i j}\right| \leq \sup \left|\left(\Psi_{\epsilon}\right)^{\prime \prime}\right|+\frac{1}{|x|}\left(\sup \left|\left(\Psi_{\epsilon}\right)^{\prime}\right|+\sup _{S^{n}}|\phi|+11 \sup _{S^{n}}|\nabla \phi|+4 \sup _{S^{n}}\left|D^{2} \phi\right|\right) .
$$

The mean curvature of $C$ is then given by

$$
S_{1}(\kappa(C))=\operatorname{div}\left(\frac{\nabla G}{\sqrt{1+|\nabla G|^{2}}}\right)=\frac{\Delta G}{\sqrt{1+|\nabla G|^{2}}}-\frac{\sum_{i j} G_{i} G_{j} G_{i j}}{\left(\sqrt{1+|\nabla G|^{2}}\right)^{3}}
$$

so that, using estimates (2.3) and (2.4), we derive the following estimate for the mean curvature of $C$ :

$$
S_{1}(\kappa(C)) \leq n \sup \left|\left(\Psi_{\epsilon}\right)^{\prime \prime}\right|+\frac{K}{|x|}
$$

for some constant $K$ depending only on $n, \sup |\phi|, \sup |\nabla \phi|, \sup \left|D^{2} \phi\right|$ and $\sup \left|\left(\Psi_{\epsilon}\right)^{\prime}\right|$. We obtain now some estimates on the function $\Psi_{\epsilon}$ and its derivatives. We may assume by construction that $\Psi_{\epsilon}$ has the particular form of a quadratic polynomial in which case we use the conditions that $\Psi_{\epsilon}(R)=0$, $\Psi_{\epsilon}\left(R-\frac{1}{2}\right)=-\epsilon$ and $\Psi_{\epsilon}(R-1)=\epsilon$, to deduce

$$
\Psi_{\epsilon}=6 \epsilon(|x|-(R-1)-1)\left(|x|-(R-1)-\frac{1}{6}\right)
$$

which yields the estimate

$$
\left|\left(\Psi_{\epsilon}\right)^{\prime}\right|+\left|\left(\Psi_{\epsilon}\right)^{\prime \prime}\right| \leq B \epsilon \leq \frac{B}{|x|}
$$

for the surface $C$ in which $|x| \in[R-1, R]$ where the constant $B$ depends only on the cone $\tilde{C}$ and the $u(0)$ estimate and the second inequality follows from Equation (2.2). Maclaurin's inequalities, together with Equations (2.5) and (2.6), then imply that

$$
S_{k}(\kappa(C)) \leq c(n, k) S_{1}^{k}(\kappa(C)) \leq \frac{B}{|x|^{k}}
$$

for some new constant $B$. We also have

$$
\psi \geq C|x|^{-\beta}
$$


so that, because $k>\beta$, for $|x|$, or equivalently $R$, sufficiently large we may assume

$$
S_{k}(\kappa(\operatorname{graph}(u)))=\psi>S_{k}(\kappa(C))
$$

over the annulus $A$. We extend $u$ to $\tilde{u}$ so that it is defined on the entire annulus $A$ by setting $\tilde{u}(x)=u(x)$ if $u$ is defined at $x$ and $\tilde{u}(x)=\partial \tilde{C}(x)$ otherwise. Note that $\tilde{u}$ is $C^{1}$. Now consider the function $d=u(x)-C(x)$ defined over that component of the region $A \cap\{x: \tilde{u}(x) \geq C(x)\}$ which intersects with the line $t \zeta$ which we denote as $\Lambda$. Clearly $d \geq 0$ on $\Lambda$. Further by the construction of $C$ it is clear that we also have $d=0$ on $\partial \Lambda$ and $\Lambda \neq \emptyset$ and $d>0$ at some point within $\Lambda$. Consider the point at which the function $d$ obtains its maximum value - at this point the principal curvatures of $C$ must exceed in magnitude the corresponding curvatures of graph $(\tilde{u})$. It is clear that this must occur at a point at which $\tilde{u}=u$ so we have the principal curvatures of $C$ exceeding in magnitude the corresponding curvatures of $\operatorname{graph}(u)$ at this point. Provided $R$ is sufficiently large this is a contradiction with Equation (2.8).

We consider now the case when $\Omega^{*}$ is unbounded and demonstrate that this is not possible. The proof is similar. We consider, for any $R$, the annulus $A_{R}=\{x: R \leq|x| \leq 2 R\}$. We then consider a surface $C_{R}$ defined over $A_{R}$ given as the graph of

$$
G=|x| \phi\left(\frac{x}{|x|}\right)+\Psi_{R}(|x|),
$$

where now

$$
\Psi_{R}(|x|)=\frac{4 C_{0}(|x|-3 / 2 R)^{2}}{R^{2}},
$$

where $C_{0}$ is the bound on $u(0)$. Direct calculation then yields the estimate

$$
\left|\Psi_{R}^{\prime}(|x|)\right|+\left|\Psi_{R}^{\prime \prime}(|x|)\right| \leq \frac{C}{R}
$$

for some new constant. We hence have (2.7) holding once more. Hence we may assume by taking $R$ sufficiently large that

$$
S_{k}(\kappa(\operatorname{graph}(u)))=\psi>S_{k}(\kappa(C)
$$

over the annulus $A_{R}$. By convexity of $\operatorname{graph}(u)$ it follows that

$$
u(x)-\tilde{C}(x) \leq C_{0}
$$


for all $x$ where, once again, $C_{0}$ is the $u(0)$ estimate. We extend $u$ as before to $\tilde{u}$. We hence have $\tilde{C}(x) \geq \tilde{u}(x)$ for all $x \in \partial A_{R}$ and $\tilde{C}\left(x_{0}\right)<\tilde{u}\left(x_{0}\right)$ for some $x_{0} \in A_{R}$ so we may apply the previous arguments.

\section{The $C^{2}$ estimate}

In this section we establish the global $C^{3, \alpha}$ estimate. We note that the global $C^{3, \alpha}$ estimate follows from a global $C^{2}$ estimate and the Krylov-Evans theory for the operator $S_{n, n-k}^{\frac{1}{k}}$ by concavity and the Maclaurin inequalities. We note that having already obtained a $C^{1}$ estimate on $h$ it follows that $f(x, h, \nabla h) \leq C$ on $\Omega$ for some constant $C$ dependant only on $f, \Omega$ and the $C^{1}$ estimate. We may therefore, due to the convexity of $\Omega$, assume the existence of a function $\underline{h}$, dependant only on the $C^{1}$ estimate for $h$, such that

$$
S_{n, n-k}^{\frac{1}{k}}\left(\lambda\left(\nabla^{2} \underline{h}+\underline{h} I\right)\right) \geq f(x, h, \nabla h)
$$

for every possible $C^{3, \alpha}$ solution $h$ due to the method of construction contained in [4]. We can reduce the global $C^{2}$ estimate to a boundary estimate on $\partial \Omega$ following the computations in the paper [4]. The tangential estimates follow trivially. The mixed tangential-normal derivative estimate at the boundary follows immediately from the computations in [4] which modify the standard approach introduced in $[2,3]$. We therefore omit the proof of the mixed normal-tangential boundary estimate. The double-normal estimate at the boundary follows the computations in [14], modified so as to hold on $S^{n}$. These modifications are elementary and the proof of the doublenormal estimate is therefore omitted.

\subsection{Reduction of the global estimate to the boundary estimate}

Theorem 7. Suppose that (in addition to the lower order estimates established above) we have the estimate

$$
\left|\nabla^{2} h\right| \leq C_{1}
$$

on $\partial \Omega$ for some constant $C_{1}$, then there exists a constant $C_{2}$ depending only on $C_{1}, n, k,\|h\|_{C^{1}\left(S_{-}^{n}\right)}$ and $\underline{h}$ up to its second derivatives such that

$$
\left|\nabla^{2} h\right| \leq C_{2}
$$

holds on all of $\Omega$. 
The proof is a modification of the computations in [4].

Proof. Consider the expression

$$
W=\max _{x \in S_{-}^{n}} \max _{\zeta \in T_{x} S_{-}^{n},|\zeta|=1}\left(h+\nabla_{\zeta \zeta} h\right)+\frac{1}{2}\left(h^{2}+|\nabla h|^{2}\right)+b \eta,
$$

where $\eta$ is a $C^{2}$ function and $b$ is a positive constant to be determined. It follows that the result will hold provided one can estimate $W$. If $W$ is achieved on $\partial S_{-}^{n}$ then an estimate already follows from Equation (3.1) and the lower order estimates. We may therefore assume $W$ achieves its maximum at an interior point $x_{0} \in S_{-}^{n}$ and in the direction of some tangent vector $\zeta \in T_{x_{0}} S^{n}$. We choose a smooth, orthonormal local frame $e_{1}, \ldots, e_{n}$ about $x_{0}$ such that $\zeta=e_{1}\left(x_{0}\right)$ and $\left\{\nabla^{2} h\right\}\left(x_{0}\right)$ is diagonal. Set $\lambda_{i}=\left(\nabla_{i i} h+\right.$ $h)\left(x_{0}\right)$. Without loss of generality, we need only estimate $\lambda_{1}=\max _{i} \lambda_{i}$ from above. We define the operator $F$ by

$$
F\left(\nabla^{2} h+h I\right)=S_{n, n-k}^{1 / k}\left(\lambda\left(\nabla^{2} h+h I\right)\right)
$$

and set

$$
\begin{aligned}
F^{i j} & =\frac{\partial F}{\partial\left(\nabla_{i j} h+h \delta_{i j}\right)}, \\
F^{i j, k l} & =\frac{\partial^{2} F}{\partial\left(\nabla_{i j} h+h \delta_{i j}\right) \partial\left(\nabla_{k l} h+h \delta_{k l}\right)} .
\end{aligned}
$$

At $x_{0}$, direct calculation reveals

$$
\left(\nabla_{i 11} h+\nabla_{i} h\right)+\lambda_{i} \nabla_{i} h+b \nabla_{i} \eta=0
$$

for all $i$ and

$$
\begin{aligned}
0 \geq & \sum_{i} F^{i i}\left\{\lambda_{i} \nabla_{i i} h+\left(\nabla_{i} h\right)^{2}+\sum_{j} \nabla_{j} h \nabla_{i i j} h+b \nabla_{i j} \eta\right\} \\
& +\sum_{i} F^{i i}\left\{\nabla_{i i 11} h+\nabla_{i i} h\right\} .
\end{aligned}
$$

Since $\left(\nabla^{2} h+h I\right)$ is diagonal at $x_{0}$ it can be shown, by direct calculation, that $F^{i j}$ is also diagonal at $x_{0}$. We drop the positive term containing $\left(\nabla_{i} h\right)^{2}$ 
Surfaces of prescribed Weingarten curvature tangential to a cone 769

and rearrange to obtain

$$
\begin{aligned}
0 \geq & \sum_{i} F^{i i}\left(\nabla_{i i 11} h+\nabla_{i i} h\right)+\sum_{i} F^{i i} \lambda_{i}^{2} \\
& -h \sum_{i} F^{i i} \lambda_{i}+\sum_{i, j} F^{i i} \nabla_{j} h \nabla_{i i j} h+b \sum_{i} F^{i i} \nabla_{i i} \eta .
\end{aligned}
$$

Differentiating (1.5) twice we obtain

$$
\sum_{i} F^{i i}\left(\nabla_{j i i} h+\nabla_{j} h\right) \geq f_{p_{j}} \lambda_{j}-C
$$

for all $j$ and

$$
\begin{gathered}
\sum_{i} F^{i i}\left(\nabla_{i i 11} h+\nabla_{i i} h\right)+\sum_{i, j, k, l} F^{i j, k l}\left(\nabla_{1 i j} h+\nabla_{1} h\right)\left(\nabla_{1 k l} h+\nabla_{1} h\right) \\
\geq \sum_{j} f_{p_{j}} \nabla_{11 j} h+f_{p_{1} p_{1}} \lambda_{1}^{2}+f_{z} \lambda_{1}-C .
\end{gathered}
$$

By concavity we may ignore the term $\sum_{i, j, k, l} F^{i j, k l}\left(\nabla_{1 i j} h+\nabla_{1} h\right)\left(\nabla_{1 k l} h+\nabla_{1} h\right)$. Using (3.2) and the formula

$$
\nabla_{i j k} h-\nabla_{j i k} h=\sum_{l} R_{k i j}^{l} \nabla_{l} h,
$$

where $R$ is the curvature tensor of the sphere, we have

$$
\begin{aligned}
\sum_{i, j} F^{i i} \nabla_{j} h \nabla_{i i j} h= & \sum_{i, j} F^{i i} \nabla_{j} h\left(\nabla_{j i i} h+\nabla_{i} h\right) \\
& +\sum_{i, j, l} F^{i i} \nabla_{j} h R_{i i j}^{l} \nabla_{l} h-|\nabla h|^{2} \sum_{i} F^{i i} \\
\geq & \sum_{j} f_{p_{j}} \nabla_{j} h \lambda_{j}-C\left(1+\sum_{i} F^{i i}\right) \\
\geq & -\sum_{j} f_{p_{j}} \nabla_{11 j} h-C\left(1+\sum_{i} F^{i i}\right)-C b .
\end{aligned}
$$

Using (3.4), the formula

$$
\begin{aligned}
\nabla_{i j k l} h-\nabla_{k l i j} h= & R_{l j k}^{m} \nabla_{i m} h+\nabla_{i} R_{l j k}^{m} \nabla_{m} h+R_{l i k}^{m} \nabla_{j m} h+R_{j i k}^{m} \nabla_{l m} h \\
& +R_{j i l}^{m} \nabla_{k m} h+\nabla_{k} R_{j i l}^{m} \nabla_{m} h,
\end{aligned}
$$


where summation notation has been used, and the observation that $\sum F^{i i} \lambda_{i}=F \leq C$, we have

$$
\begin{aligned}
\sum_{i} F^{i i}\left(\nabla_{i i 11} h+\nabla_{i i} h\right)= & \sum_{i} F^{i i}\left(\nabla_{i i 11} h+\nabla_{11} h\right)+\sum_{i} F^{i i}\left(\lambda_{i}-\lambda_{1}\right) \\
\geq & \sum_{i} F^{i i}\left(\nabla_{11 i i} h+\nabla_{11} h\right)+\sum_{i} F^{i i}\left(\lambda_{i}-\lambda_{1}\right) \\
& +2 \sum_{i} F^{i i} R_{i i 1}^{1}\left(\lambda_{1}-\lambda_{i}\right)-C \sum_{i} F^{i i} \\
\geq & \lambda_{1} \sum_{i>1} F^{i i}+\sum_{j} f_{p_{j}} \nabla_{11 j} h+f_{p_{1} p_{1}} \lambda_{1}^{2} \\
& +f_{z} \lambda_{1}-C\left(1+\sum_{i} F^{i i}\right) .
\end{aligned}
$$

From (3.3), (3.5) and (3.6) we obtain

$$
\lambda_{1}\left(\sum_{i>1} F^{i i}+f_{z}\right)+f_{p_{1} p_{1}} \lambda_{1}^{2}+b \sum_{i} F^{i i} \nabla_{i i} \eta \leq C(1+b)+C \sum_{i} F^{i i} .
$$

We now observe that

$$
\sum_{i} F^{i i} \nabla_{i i}(\underline{h}-h) \geq \epsilon \sum_{i} F^{i i}-C
$$

for some uniform constant $\epsilon>0$. This follows directly from the concavity of $F$. Writing $w=\underline{h}-\epsilon$, where $\epsilon$ is chosen sufficiently small to ensure that $w$ is still admissible in the sense that $\left(\nabla^{2} w+w I\right)>0$ on $\Omega$, concavity of $F$ implies

$$
F\left(\nabla^{2} w+w I\right) \leq F\left(\nabla^{2} h+h I\right)+F^{i j}\left(\left(w_{i j}-h_{i j}\right)+\delta_{i j}(w-h)\right) .
$$

Rearranging we derive (3.8). Setting $\eta=\underline{h}-h$, Equation (3.8) implies

$$
\sum_{i} F^{i i} \nabla_{i i} \eta \geq \epsilon \sum_{i} F^{i i}-C
$$

for some uniform constant $\epsilon>0$. We may hence select $b$ sufficiently large to derive, from (3.7),

$$
\lambda_{1}\left(\sum_{i>1} F^{i i}+f_{z}\right)+f_{p_{1} p_{1}} \lambda_{1}^{2} \leq C .
$$


We observe that the matrix $f_{x_{i} x_{j}}$ (where $x_{i}$ are now the coordinates of the Euclidean space $\mathbb{R}^{n+1}$ in which the sphere $S^{n}$ is embedded) at the point $x_{0}$ is of the form

$$
\left(f_{x_{i} x_{j}}\right)=\left(\begin{array}{cc}
A & \alpha \\
\beta & \gamma
\end{array}\right)
$$

for some constants $\alpha, \beta$ and $\gamma$ where $A$ is an $n \times n$ matrix whose eigenvalues are bounded below by some positive constant $\delta_{1}>0$ which depends only on the strict convexity of $\psi^{-1 / k}$ in $x$. Furthermore, all the eigenvalues of the matrix $f_{x_{i} x_{j}}$ are non-negative by the convexity of $\psi^{-1 / k}$ in $(x, u)$. Since the vector $p_{1}$ lies in the tangent space to $S^{n}$ at $x_{0}$, and since $\Omega \subset \subset S_{-}^{n}$ it follows that the magnitude of the projection of $p_{1}$ onto the plane formed by the first $n$ coordinates $x_{1}, \ldots, x_{n}$ is bounded below by some constant $\delta_{2}>0$ depending only on $\operatorname{dist}\left(\Omega, \partial S_{-}^{n}\right)$. Therefore, we deduce that $f_{p_{1} p_{1}}=$ $p_{1}^{T}\left(f_{x_{i} x_{j}}\right) p_{1}>\delta$ for some $\delta>0$ depending only on $\delta_{1}$ and $\delta_{2}$. Together with (3.9) this demonstrates the bound

$$
\lambda_{1} \leq C
$$

and the proof is complete.

\section{Existence}

Once the global $C^{2}$ estimate has been established Equation (1.5) becomes uniformly concave (see [18]) and so the Krylov-Evans regularity theory applies and we immediately obtain a global $C^{3, \alpha}$ estimate for $h$ and hence one can apply the continuity method and existence is established. The proof of Theorems 2 and 3 is therefore complete. We now provide the proof of Theorem 4.

Proof of Theorem 4. Fix any supposed solution $u$. Let us define the perturbed cone $\tilde{C}_{\epsilon, \delta}$ for $\epsilon>0, \delta>0$ derived from our original cone $\tilde{C}$ by setting

$$
\tilde{C}_{\epsilon, \delta}(x)=(1-\delta) \tilde{C}(x)+\epsilon
$$

We then define

$$
\delta(\epsilon)=\sup \left\{\delta \geq 0: \tilde{C}_{\epsilon, \delta} \cap \operatorname{graph}(u) \neq \emptyset\right\}
$$

It is clear that $\delta(\epsilon)>0$ for all $\epsilon$ and $\delta(\epsilon)<\infty$ for all $\epsilon$ sufficiently small. We also have $\delta(\epsilon) \rightarrow 0$ as $\epsilon \rightarrow 0$. To see that this is the case observe that 
we have

$$
\tilde{C}_{\epsilon, \delta} \cap \tilde{C}=\tilde{C} \cap\left\{x_{n+1}=\frac{\epsilon}{\delta}\right\}
$$

which can be obtained by noting that at the points at which $\tilde{C}_{\epsilon, \delta}$ and $\tilde{C}$ intersect we have

$$
(1-\delta) \tilde{C}+\epsilon=\tilde{C}(x)
$$

upon which (4.1) follows by a simple rearrangement. We then note that if

$$
\frac{\epsilon}{\delta}<\frac{\inf u}{2}
$$

then the set of points at which $\tilde{C}_{\epsilon, \delta}$ and $\tilde{C}$ intersect lie strictly below inf $u$ and hence strictly below the surface $\operatorname{graph}(u)$. It hence follows that $\tilde{C}_{\epsilon, \delta} \cap$ $\operatorname{graph}(u)=\emptyset$. Rearranging (4.2) and noting the definition of $\delta(\epsilon)$ we hence obtain the bound

$$
\delta(\epsilon) \leq \frac{2 \epsilon}{\inf u}
$$

so that (as we must have inf $u>0$ ) it indeed holds that $\delta(\epsilon) \rightarrow 0$ as $\epsilon \rightarrow$ 0 although the rate of this convergence may depend upon the particular solution $u$. We observe that the curvature of the cone $\tilde{C}_{\epsilon, \delta}$ satisfies

$$
S_{k}\left(\kappa\left(\tilde{C}_{\epsilon, \delta}(x)\right)\right)=|x|^{-k} S_{k}\left(\kappa\left(\tilde{C}_{\epsilon, \delta}\left(\frac{x}{|x|}\right)\right)\right)
$$

which is independent of $\epsilon$. For any fixed $\eta \in S^{n-1}$ it follows from direct calculation that

$$
\lim _{\delta \rightarrow 0} S_{k}\left(\kappa\left(\tilde{C}_{\epsilon, \delta}(\eta)\right)\right)=S_{k}(\kappa(\tilde{C}(\eta)))
$$

so that, as the set $S^{n-1}$ is closed, we have

$$
\lim _{\epsilon \rightarrow 0} \sup _{\eta \in S^{n-1}}\left|S_{k}\left(\kappa\left(\tilde{C}_{\epsilon, \delta(\epsilon)}(\eta)\right)\right)-S_{k}(\kappa(\tilde{C}(\eta)))\right|=0
$$

Now consider for any fixed $\epsilon<\frac{\inf u}{2}$ the set of points

$$
X_{\epsilon}:=\tilde{C}_{\epsilon, \delta(\epsilon)} \cap \operatorname{graph}(u)
$$

which is not empty by the definition of $\delta(\epsilon)$. Defining the distance function

$$
d_{\epsilon}(x)=\operatorname{graph}(u)(x)-\tilde{C}_{\epsilon, \delta(\epsilon)}(x)
$$


it follows that there exists at least one point $x_{0}=x_{0}(\epsilon) \in X_{\epsilon}$ such that $d_{\epsilon}\left(x_{0}\right)=0$. Furthermore it is clear, by construction, that $d_{\epsilon}(x) \geq 0$ for all $x$. We apply the maximum principle at the point $x_{0}$ to determine

$$
\begin{aligned}
C_{0}\left|x_{0}\right|^{-k} & \geq \psi\left(x_{0}, u\left(x_{0}\right), \nu\left(x_{0}\right)\right)=S_{k}\left(\kappa\left(\operatorname{graph}(u)\left(x_{0}\right)\right)\right) \\
& \geq S_{k}\left(\kappa\left(\tilde{C}_{\epsilon, \delta(\epsilon)}\left(x_{0}\right)\right)\right)=\left|x_{0}\right|^{-k} S_{k}\left(\kappa\left(\tilde{C}_{\epsilon, \delta(\epsilon)}\left(\frac{x_{0}}{\left|x_{0}\right|}\right)\right)\right) .
\end{aligned}
$$

By taking $\epsilon$ sufficiently small and using Equation (4.3) we may write

$$
C_{0}\left|x_{0}\right|^{-k} \geq \frac{3}{4} \inf _{\eta \in S^{n-1}} S_{k}(\kappa(\tilde{C}(\eta)))\left|x_{0}\right|^{-k}
$$

so that selecting $C_{0}=\frac{1}{2} \inf _{\eta \in S^{n-1}} S_{k}(\kappa(\tilde{C}(\eta)))$ we derive a contradiction and determine that no solution, either bounded or unbounded, can exist.

We now prove Theorem 5:

Proof of Theorem 5. The main idea is to make use of the formulae established in [9] which, while highly technical in the general case, simplify considerably in the rotationally symmetric case. In particular from [9] for a rotationally symmetric solution $u$, which we shall write as $u(r)$, to Problem 1 , we have

$$
\begin{aligned}
k \int_{B_{R}} \psi & =\left(\frac{\left|u^{\prime}(R)\right|}{\sqrt{1+\left|u^{\prime}(R)\right|^{2}}}\right)^{k} \int_{\partial B_{R}} S_{k-1}\left(\kappa\left(\partial B_{R}\right)\right) \\
& =\left(\frac{\left|u^{\prime}(R)\right|}{\sqrt{1+\left|u^{\prime}(R)\right|^{2}}}\right)^{k} n \omega(n)\left(\begin{array}{c}
n-1 \\
k-1
\end{array}\right) R^{n-k}
\end{aligned}
$$

for all $R>0$ for which $u$ is defined. Here $\omega(n)$ is the volume of the $n$ dimensional unit ball. The boundary condition then gives us that the domain of definition of our radially symmetric solution $u$ is $\Omega^{*}=B_{R^{*}}$ where $R^{*}$ is the smallest $R>0$ for which

$$
k \int_{0}^{R^{*}} r^{n-1} \psi(r) d r=\left(\frac{K}{\sqrt{1+K^{2}}}\right)^{k}\left(\begin{array}{l}
n-1 \\
k-1
\end{array}\right)\left(R^{*}\right)^{n-k}
$$

holds. We hence define the function $d$ as in Equation (1.8) and determine that $d\left(R^{*}\right)=0$ and $d(R)<0$ for $0<R<R^{*}$ is a necessary condition for the existence of a bounded solution of radius $R^{*}$. We demonstrate that it is also 
sufficient. Consider solving the Dirichlet problem

$$
S_{k}(\kappa(\operatorname{graph}(u)))=\psi
$$

on the ball $B_{R^{*}}$ subject to the boundary condition $u \equiv 0$ on $\partial B_{R^{*}}$. The estimates from Sections 2 and 3 no longer apply as we no longer assume $\psi^{-1 / k}$ is convex. However, the required estimates may be determined directly by considering the ODE corresponding to (4.5). Existence then follows from the standard arguments while formula (4.4) implies that $u^{\prime}\left(R^{*}\right)=K$ so that $u$ is a solution to our original problem after a suitable translation in the $x_{n+1}$ direction. For the complete non-compact case we observe that it is possible to rewrite the function $g(s)$ as

$$
g(s)=\frac{\left(\left(\frac{K}{\sqrt{1+K^{2}}}\right)^{k}+\frac{d(s)}{\left(\begin{array}{l}
n-1 \\
k-1
\end{array}\right) s^{n-k}}\right)^{1 / k}}{\left(1-\left(\left(\frac{K}{\sqrt{1+K^{2}}}\right)^{k}+\frac{d(s)}{\left(\begin{array}{l}
n-1 \\
k-1
\end{array}\right) t^{n-k}}\right)^{2 / k}\right)^{1 / 2}}
$$

so that Equation (1.9) implies that $d(R) R^{k-n} \rightarrow 0$ as $R \rightarrow \infty$. By the definition of the function $d$ this implies the limit

$$
\lim _{R \rightarrow \infty} \frac{k \int_{0}^{R} r^{n-1} \psi(r) d r}{\left(\frac{K}{\sqrt{1+K^{2}}}\right)^{k}\left(\begin{array}{l}
n-1 \\
k-1
\end{array}\right) R^{n-k}}=1 .
$$

Hence for any $\delta>0$ there exists an $R^{*}(\delta)<\infty$ such that

$$
k \int_{0}^{R^{*}(\delta)} r^{n-1}(1+\delta) \psi(r) d r=\left(\frac{K}{\sqrt{1+K^{2}}}\right)^{k}\left(\begin{array}{l}
n-1 \\
k-1
\end{array}\right)\left(R^{*}(\delta)\right)^{n-k}
$$

We may therefore determine, by the previous reasoning, that for any $\delta>0$ there exists a solution $u_{\delta}$ to Problem 1 with right-hand side given by $(1+\delta) \psi$ defined over the ball $B_{R^{*}(\delta)}$ where $R^{*}(\delta) \rightarrow \infty$ as $\delta \rightarrow 0$. We translate each solution so that $u_{\delta}(0)=0$ for all $\delta$. A uniform $C^{1}$ estimate on $u_{\delta}$ on compact subsets then follows using the formulae in [9] while a uniform estimate of the form $\left\|u_{\delta}\right\|_{C^{2, \alpha}\left(B_{R}\right)} \leq C(R)$ follows from the interior curvature estimate in [11]. By taking the limit of the sequence $u_{\delta}$ on compact subsets we hence deduce the existence of a function $u$ whose graph is a $k$-convex complete noncompact hypersurface of curvature $\psi$ and it remains only to demonstrate 
that $\operatorname{graph}(u)$ is asymptotically tangential to the cone $\tilde{C}$. It is clear that $u$ must be radially symmetric and so we shall simply write $u=u(r)$. Let us consider the function

$$
\rho(t)=u(t)-K t
$$

which is non-increasing and measures the signed distance between the line of slope $K$ passing through the origin and the surface graph $(u)$. As $\rho(0)=0$ we may write

$$
\rho(t)=\int_{0}^{t}\left(u^{\prime}(s)-K\right) d s .
$$

Using Equation (4.4) we may rewrite this integral as the one given in Equation (1.9). It hence follows that if Equation (1.9) holds then

$$
\sup |u(t)-K t| \leq C
$$

for some constant $C$. Therefore

$$
u(t)>K t-2 C
$$

for all $t \geq 0$. We then define $C^{*}<\infty$ by

$$
C^{*}=\inf \{C \geq 0: u(t) \geq K t-C \forall t \geq 0\}
$$

It is clear that $u(t) \geq K t-C^{*}$ for all $t$. We also claim that $g(t)=u(t)-$ $\left(K t-C^{*}\right) \rightarrow 0$ as $t \rightarrow \infty$. Together with the observation that $u^{\prime}(t) \rightarrow K$ as $t \rightarrow \infty$ this is sufficient to demonstrate that $u+C^{*}$ is the desired complete, non-compact solution to Problem 1. Indeed $g(0)=C^{*}$ and $g$ is a strictly decreasing positive function hence there exists $\delta \geq 0$ such that $g(t) \rightarrow \delta$ as $t \rightarrow \infty$ and it remains only to show that $\delta=0$. The function $g$ is strictly decreasing because $u^{\prime}(t)<K$ for all $t$ which follows from the assumption that $d(t)<0$ for all $t$. If $\delta>0$ then we have $u(t) \geq K t-\left(C^{*}-\delta / 2\right)$ which contradicts with the definition of $C^{*}$. We now prove, continuing under the assumption that $d<0$ everywhere, that if Equation (1.9) does not hold then there cannot exist a rotationally symmetric complete solution to Problem 1. Suppose there existed a rotationally symmetric solution $u$ to Problem 1 then the signed distance between the solution $u$ and the cone $\tilde{C}$ at radius $R$ would 
be given by

$$
u(0)+\int_{0}^{\infty}\left(\frac{\left(k \int_{0}^{s} r^{n-1} \psi(r) d r\left(\begin{array}{l}
n-1 \\
k-1
\end{array}\right)^{-1} s^{k-n}\right)^{1 / k}}{\left(1-\left(k \int_{0}^{s} r^{n-1} \psi(r) d r\left(\begin{array}{l}
n-1 \\
k-1
\end{array}\right)^{-1} s^{k-n}\right)^{2 / k}\right)^{1 / 2}}-K\right) d t
$$

This integral diverges to $-\infty$ so it is clear that the solution cannot lie entirely within any cone of slope $K$ and therefore the boundary condition cannot be satisfied. The observation that $u$ is convex if and only if the function $g(s)$ in Theorem 5 is non-decreasing follows immediately from the identification of this term with $u^{\prime}(s)$.

\section{References}

[1] A.D. Alexandrov, Zur theorie der gemischten volumina von konvexen korpern, iii. die erweiterung zweeier lehrsatze minkowskis uber die konvexen polyeder auf beliebige konvexe flachen, Mat. Sbornik N.S. 3 (1938), 27-46.

[2] L. Caffarelli, L. Nirenberg and J. Spruck, The Dirichlet problem for nonlinear second-order elliptic equations. I. Monge-Ampere equation, Comm. Pure Appl. Math 37(3) (1984), 369-402.

[3] B. Guan, The Dirichlet problem for a class of fully nonlinear elliptic equations, Comm. Partial Differential Equations 19(3-4) (1994), 399-416.

[4] B. Guan, The Dirichlet problem for Hessian equations on Riemannian manifolds, Calc. Var. Partial Differential Equations 8(1), (1999), 45-69.

[5] P. Guan, C. Lin and X.-N. Ma, The Christoffel-Minkowski problem. II. Weingarten curvature equations, Chinese Ann. Math. Ser. B 27(6) (2006), 595-614.

[6] P. Guan, X-N. Ma and F. Zhou, The Christoffel-Minkowski problem. III: Existence and convexity of admissible solutions, Comm. Pure Appl. Math 59(9) (2006), 1352-1376.

[7] J.N. Holland, An extremal case of the equation of prescribed Weingarten curvature, Calc. Var. Partial Differential Equations 48 (2012), 277-291. 
Surfaces of prescribed Weingarten curvature tangential to a cone 777

[8] P.L. Lions, N.S. Trudinger and J.I.E. Urbas, The Neumann problem for equations of Monge-Ampere type, Comm. Pure Appl. Math. 39(4) (1986), 539-563.

[9] R. Reilly, On the Hessian of a function and the curvatures of its graph, Michigan Math. J. 20 (1973), 373-383.

[10] W. Sheng, N.S. Trudinger and X-J. Wang, Convex hypersurfaces of prescribed Weingarten curvatures, Comm. Anal. Geom. 12(1-2) (2004), 213-232.

[11] W. Sheng, J.I.E. Urbas and X.-J. Wang, Interior curvature bounds for a class of curvature equations, Duke Math. J. 123(2) (2004), 235-264.

[12] N. Stavrou, Selfsimilar solutions to the mean curvature flow, J. Reine Angew. Math. 499 (1998), 189-198.

[13] N.S. Trudinger, A priori bounds and necessary conditions for solvability of prescribed curvature equations, Manuscripta Math. 67(1) (1990), 99-112.

[14] N.S. Trudinger, On the Dirichlet problem for Hessian equations, Acta Math. 175(2) (1995), 151-164.

[15] J.I.E. Urbas, textitThe equation of prescribed Gauss curvature without boundary onditions, J. Differential Geom. 20(2) (1984), 311-327.

[16] J.I.E. Urbas, Complete noncompact self-similar solutions of Gauss curvature flows. I. Positive powers, Math. Ann. 311(2) (1998), 251-274.

[17] J.I.E. Urbas, Complete noncompact self-similar solutions of Gauss curvature flows. II. Negative powers, Adv. Differential Equations 4(3) (1999), 323-346.

[18] X.-J. Wang, The k-Hessian equation, in 'Geometric analysis and PDEs', eds. S.-Y.A. Chang, A. Ambrosetti and A. Malchiodi, Lect. Notes Maths., Springer, Dordrecht, 2009, 177-252.

4 Lorikeet Close

Fig Tree Pocket

BRISBANE 4069

QUEENSLAND

Australia

E-mail address: james.holland@uqconnect.edu.au

ReCeived June 5, 2012 
